\title{
Nutritional Considerations in Childhood Acute Lymphoblastic Leukemia
}

\author{
Sami El Khatib ${ }^{1,2, *}$, Mona Omar ${ }^{2}$ \\ ${ }^{1}$ Department of Biomedical Sciences, School of Arts \& Sciences, Lebanese International University, Lebanon \\ ${ }^{2}$ Department of Biological Sciences, School of Arts \& Sciences, Lebanese International University, Lebanon
}

Received December 8, 2019; Revised January 19, 2020; Accepted February 7, 2020

Copyright $\bigcirc 2020$ by authors, all rights reserved. Authors agree that this article remains permanently open access under the terms of the Creative Commons Attribution License 4.0 International License

\begin{abstract}
The high prevalence of malnutrition in adult and pediatric cancer patients has been appreciated for decades and continues to be documented. Although the prognostic significance of nutritional status among patients with cancer remains controversial, it is generally accepted that the nutritional support is an important as pect of medical therapy. Children with cancer are particularly vulnerable to malnutrition, because they exhibit elevated substrate needs due to the disease and its treatment. At the same time, children have increased requirements of nutrients to attain appropriate growth and neurodevelopment. Malnutrition in children with cancer should not be accepted at any stage of the disease or tolerated as an inevitable process. The undernourished patients of ALL are more prone to infections, requiring longer duration of hospital stay. Therefore, optimum nutritional support to such patients can help to decrease the chances of infections and ultimately improve the outcome based on a systematic assessment. Nutritional assessment is the first step of nutritional support which will not improve the child's sense of well-being but also maintain normal growth, promote wound healing, improve tumor response to therapy and enable the child to better withstand the effects of therapy. Assessment will also help prevent over nutrition which may in some instances be detrimental to the treatment of cancer. Therefore, nutritional adequate strategies should be considered and integrated as a fundamental feature of pediatric oncology with the same diligence as one does for other supportive care measures to prevent chronic illness and adverse late effects caused by malnutrition in children with ALL.
\end{abstract}

Keywords Pediatric Nutrition, Acute Lymphoblastic Leukemia, Malnutrition, Oncology, Childhood ALL

\section{Introduction}

Cancer also termed malignancy is an abnormal growth of cells. There are more than 100 type of cancerincluding breast cancer, skin cancer, lung cancer, colon cancer, prostate cancer, and lymphoma. Symptoms vary on the type. The treatment may include chemotherapy, radiation, and surgery. Many search articles and clinical trials were conducted under the title of oncology aiming at exploring cancer cell behavior and discovering the most optimal cure. It's every researcher's dream. This research investigates the nutritional role or in other words its impact on cancer growth and prognosis aside by the conventional treatment. The current review shed the light over the nutritional management effect on patients with cancer during therapy and afterward. The manuscript includes firstly an overview about the most relevant type of childhood cancer which is leukemia and indicating the optional treatments. We thereafter incorporate few nutrition considerations as malnutrition effect on treatment results, serum zinc and copper role in malignancy, supplement of folate and $\mathrm{B}_{12}$ in deficient patients, mucositis and sinusoidal obstructive syndrome, drug and nutrient interaction, and oxidative stress. Glutamine, antioxidant, and other dietary supplements that might lift up the treatment overall outcome with less side effects are then highlighted. Nutrition ingredients are becoming the new breakthrough in medical remedies as sulforaphane and garlic compounds among the adopted nutritional recommendations pinpointed hereby.

\section{Acute Lymphoblastic Leukemia and Treatment Options}

\subsection{Overview of Leukemia}

Cancer is a disorder in cells regulatory system and development leading to the formation of abnormal cell division and production. Cancer can almost start anywhere in the human body. These unregulated cells may spread to other organs and tissues, thus turning into cancer or become situational tumor (Owens, 2013). 


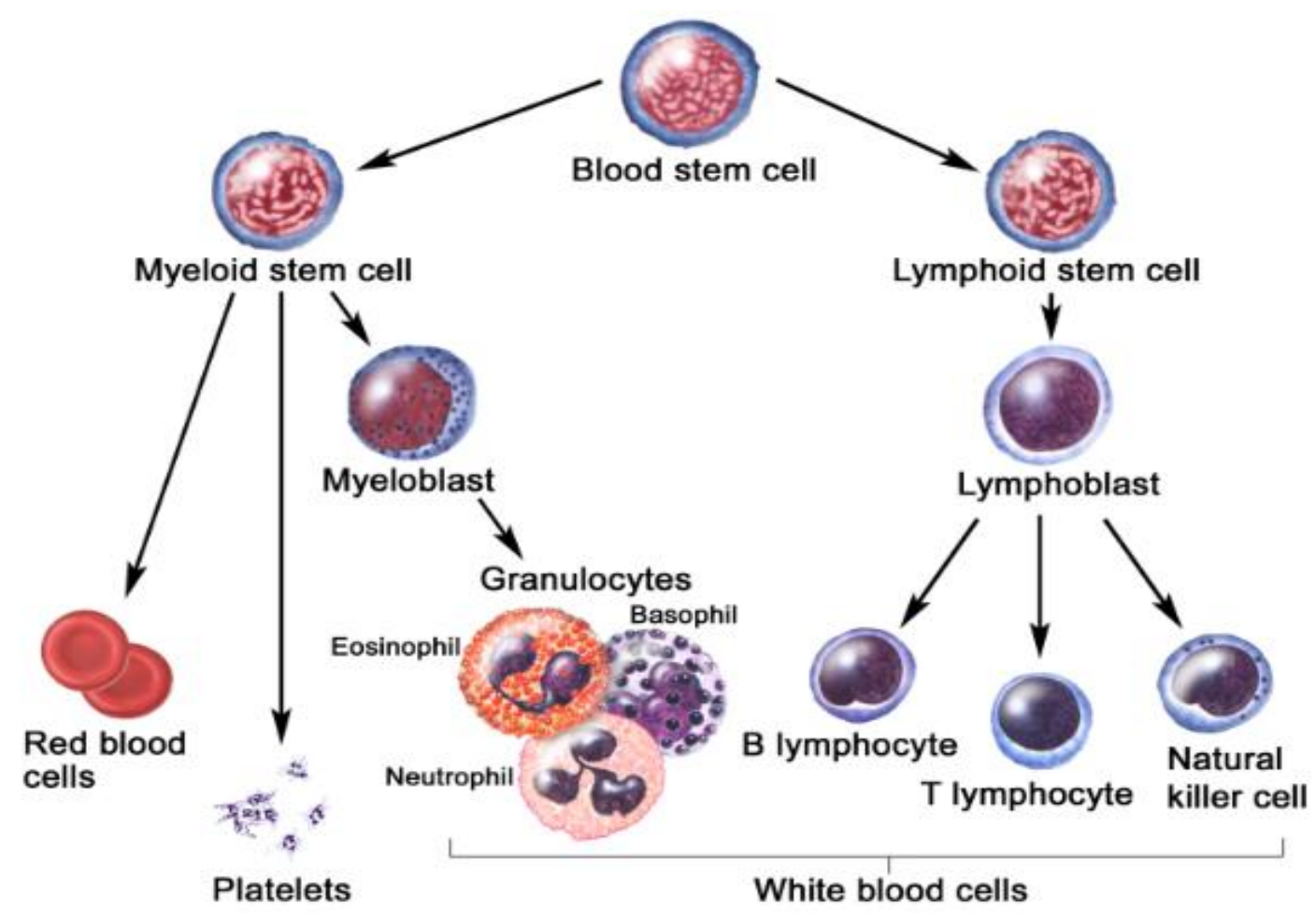

Figure 1. Blood cell development. A blood stem cell goes through several steps to become a red blood cell, platelet, or white blood cell (PDQ, 2016)

Leukemia is one of the many forms that cancer might take. It originates in the bone marrow and liberates to the blood steam. In a healthy child, the bone marrow produces blood stem cell (immature cells) that becomes mature over time. These stem cells may assume a form of myeloid stemcell or a lymphoid stem cell. Myeloid stem cells are transformed to either one of the three types of mature blood cells:

- Red blood cells (RBC) that is responsible of transporting oxygen to other substances to all body tissues and organs.

- Platelets, which forms blood clots in order to cause bleeding.

- White blood cells (WBC), which partition in bodies defense against infections and diseases. They are monocytes and granulocytes (eosinophils, basophils, and neutrophils), as demonstrated in figure 1 .

A lymphoid stem cells turns into a lymphoblast cell, then into lymphocyte (WBC):

- B lymphocytes, that produces antibodies

- T lymphocytes, that acids B lymphocytes to make antibodies for fighting infections

- Natural killer cells (NK cells), that attack tumor cells and virus-infected cells (PDQ, 2016)

Leukemia cells are a term given to cancerous cells produced from either myeloid or lymphoid cell lines. This term is later divided into acute (rapid onset and progression) or chronic (slow onset and progression) malignant cells. It is further sub-divided to lymphoid (lymphocytic or lymphoblastic) or myeloid cells. Chronic lymphocytic leukemia, chronic myeloid leukemia, acute lymphocytic leukemia (ALL), acute myeloid leukemia are common types of leukemia of which ALL is the most common type of cancer in children (Owens, 2013).

\subsubsection{Incidence of ALL}

White children ALL incidences are approximately three times higher than in Black children from ages 2 to 3 years old. Meanwhile the overall incidence of ALL has been increasing slowly in the past decades. For instance, it is nearly about 30 to 40 case in every 1 million in pediatrics under the age of 20 years old in the United States (Owens, 2013). Each year relatively 3100 US people who are under the age of 20 are diagnosed with ALL. It is greater seen in who are 10 years old and above than in infants and 8 years old (PDQ, 2016). A 34 incident in every 1 million shows to have ALL in His panic children as it appears to be the greatest incidence (PDQ, 2016).

\subsubsection{Causes and Risk Factors}

There is no one causative factor has been associated or link to cancer development; meanwhile some risk factors are identified to increase of ALL risk. Some of them include the following:

- Prenatal exposure to X-rays.

- Exposure to radiation, such as therapeutic radiation used for thymus enlargement for example.

- Past chemotherapy treatment.

- Genetic conditions, that can be detected in $75 \%$ to $80 \%$ of all childhood ALL (Owens, 2013), as: 
- Down syndrome, also known as trisomy 21, a condition in which extra genetic material cause delays in child's development mentally and physically.

- Neurofibromatosis (NFI mutation) a genetic disorder in which neurofibromas from throughout nervous system.

- Blood syndrome (BLM gene mutation), an inherited disorder characterized by short stature a skin rash that develops after exposure to the sun and greatly increased risk of cancer.

- Ataxia-telangiectasia (ATM gene mutation), refers to as Louis-bar syndrome, a rare neurodegenerative autosomal recessive disease causing severs disability. Ataxia refers to poor coordination and telangiectasia is a small dilated blood vessel(hall marks of disease). Li-Frawmeni syndrome, constitutional mismatch repair deficiency, low-and-high-penetrance inherited genetic variants, and Fanconi anemia are other genetic disorders (PDQ, 2016).

\subsubsection{Signs and Symptoms}

The increasing number of leukemia cells in blood and bone marrow leads to a narrower spaces left for WBC, RBC, and platelets causing infections, anemia, and bleeding. Other signs and symptoms include malaise, lymph node enlargement, bone or joint pain, fever, pallor, loss of appetite, petechial, abdominal discomfort (pain, feeling of fullness below ribs), and swelling (Owens, 2013).

\subsubsection{Diagnosis}

Tests and procedures are used to distinguish childhood ALL, and to check if cancer cells have spread to other body organs or tissues. The diagnosis includes a physical examination to assess lumps existence or anything else that seems unusual. Checking the medical history of the patient also comes in favor. A complete blood count (CBC) where a blood sample is drawn and examined for any abnormality in number of RBC, platelets, and WBC with these types, as well for anemia checking. Measuring blood chemistry for specific substances released by some organs into the blood and comparing them to usual concentrations for detection of certain diseases. Even with the enhanced production of undifferentiated blast cells, the main confirmation for the occupation of leukemia cells in the bone marrow is through completion of a bone marrow aspiration or biopsy. Lumbar puncture and chromosomal analysis are used to specify the type of leukemia (Owens, 2013).

\subsection{Chemotherapy}

Before tackling the treatment options of children with ALL, the treating group of experts must put into consideration the child age, gender, race which WBC leukemia has originated from as well any genetic changes in lymphocytes whether the child has a standard-risk (with
$\mathrm{WBC}$ count $<50000 / \mu \mathrm{l}$ at diagnosis) or a high-risk (WBC count $\geq 50000 / \mu \mathrm{l}$ at diagnosis). In addition of assuming of what is prognosis might be like. With that being said the team; entrusted group to treat and follow-up disease progression and outcome; incorporates all the necessary skills and assistance from the health care professionals to ensure that patients are receiving the right treatment, care and rehabilitation to achieve optimal survival and quality of life (PDQ, 2016; PDQ patient's overview, 2016).

Phases of therapy are divided into:

1. Remission induction phase.

2. Consolidation/ intensification phase.

3. Maintenance or continuation phase (PDQ, 2016; PDQ patient's overview, 2016).

\subsubsection{Induction Phases}

In remission induction chemotherapy, the first phase of treatment of a 4-week duration. Its main goal is to abet a full remission. This is accomplished by using drugs to kill leukemia cells wherever they exist in the body. This phase typically consists of the following drugs with or without anthracycline (daunorubian or doxorubicin):

- Vincristine, a plant alkaloid used in the management of leukemia and lymphomas. It is an anti-cancer (antineoplastic or cytotoxic).

- Corticosteroid, either prednisone or dexamethasone, were dexamethasone has more favorable outcomes. Acting as anti-inflammatory, anti-neoplastic and immunosuppressive.

- L-asparaginase, which is classified as an enzyme and several forms like pegaspargase (PEG-asparaginase), Erwiana L-asparaginase, and native Escherichia coli Lasparaginase are used in the treatment. Putting into consideration those patients who are allergic to pegaspargase be compelled to use Erwiana Lasparaginase (PDQ, 2016).

\subsubsection{Consolidation \& Intensification Phase (Therapy)}

This second phase of treatment aims at eliminating any remaining leukemia cells in the body to avoid relapse. It begins once the leukemia is in remission and in conjunction with central nervous system-directed therapy. The risk group implies the intensity of post induction therapy (PDQ, 2016). BFM (Berlin Frankfurt Munster) backbone is the most adopted intensification schema, and it includes the following:

1. Initial consolidation, immediately following the complete remission phases. Where cyclophosphamide, low-dose without leucovorin rescue are being used and mercaptopurine are used.

2. Interim maintenance phases, where multiple doses of high-doses methotrexate $\left(5 \mathrm{~g} / \mathrm{m}^{2}\right)$ with leucovorin rescue or escalating doses of methotrexate (starting dose $100 \mathrm{mg} / \mathrm{m}^{2}$ ) without leucovorin rescue are being used.

3. Reduction, which follows a similar order of drugs and timing that is used during induction and initial consolidation rounds. 
4. Maintenance, of daily mercaptopurine a weekly lowdose methotrexate and sometimes administration of vincristine and a corticosteroid, as well as continued intrathecal therapy (PDQ, 2016).

\subsubsection{Maintenance Phase}

The third phase shoots at extermination of leukemia cells that may have survived the previous phases of treatments to avoid risk of relapse. Generally, maintenance chemotherapy continues 2 to 3 years along with cranial radiation (CR) continuation. Extending it beyond 3 years has no favorable outcomes. A standard protocol accommodates daily oral mercaptopurine and weekly oral or parenteral methotrexate, nevertheless non-compliance of adherence to these lessened drug dosages are as sociated with significant risk of relapse. Therefore, a careful monitoring of children by the medical team is critical at all times (PDQ, 2016).

The administration of drugs can be done by two means, a mouth or vein. If the chemotherapeutic drug or drugs are directly administered into the target area where leukemia cells are located it's then called regional chemotherapy, such as intrathecal chemotherapy. Meanwhile allocating drugs into the blood stream spreading throughout the body to reach its target cells is systemic chemotherapy. Putting into consideration that high-risk ALL children acquire more intensified therapy where they include more anti-cancer drugs with high doses than low-risk ALL patients (PDQ patient's overview, 2016).

\subsection{CNS-Directed Therapy for Childhood ALL}

Almost $3 \%$ of children appear to have central nervous system 3 (CNS3) diseases upon diagnosis. CNS3 is noted by the detection of 5 or more $\mathrm{WBC}$ found in the cerebrospinal fluid (CNF) specimen in addition to presence or absence of cranial nerve palsies. An overt-CNS leukemia will flourish in most children where or not lymphoblast was detected in the spinal fluid at primary diagnosis, except a specific therapy is conducted towards the CNS. Consequently, ALL are to receive a combination therapy with CNS prophylaxis. Due to the fact that CNS is sanctuary early directed treatment are launched early to eliminate clinically evident CNS diseases upon diagnosis and to baffle CNS relapse in all patients (PDQ, 2016).

Standard treatment options include:

1. Intrathecal chemotherapy: usually starts at the beginning of induction phases, and continuous throughout maintenance phases but with variable intensities. It may use methotrexate alone or in addition with cytarabine and hydrocortisone forming triple intrathecal chemotherapy. Intrathecal methotrexate has significant systemic effect that contributes to marrow relapse prevention may have been noticed.

2. CNS-directed systemic chemotherapy: it systemically delivers agents that own some properties. The following drugs include dexamethasone, Lasparaginase, high dose methotrexate with leucovirin rescue, escalating IV methotrexate dose without leucovirin rescue.

3. Cranial improvement: a high energy x-rays or gamma rays are used to destroy leukemia cells. It's used for special cases and high risk CNS leukemia.

A dramatic improvement was shown as CNS-directed therapies were combined with treatment regimens. Usually intrathecal chemotherapy combined with CNS-directed systemic chemotherapy is standard and commonly used for newly diagnosed ALL children as presented in table 1. As for cranial radiation it is selective (PDQ, 2016).

Table 1. CNS-Directed Treatment Regimens for Newly Diagnosed Childhood ALL (PDQ, 2016)

\begin{tabular}{|c|c|c|}
\hline Standard Tre atment Options & Standard-risk ALL Patient & High-risk ALL Patient \\
\hline In trathecal chemotherapy & 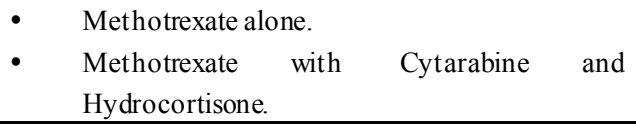 & $\begin{array}{l}\text { - } \quad \text { Methotrexate alone. } \\
\text { Methotrexate with Cytarabine and } \\
\text { Hydrocortisone. }\end{array}$ \\
\hline $\begin{array}{l}\text { CNS-directed systemic } \\
\text { chemotherapy }\end{array}$ & $\begin{array}{l}\text { - } \\
\text { - } \quad \text { Lexamethanose. } \\
\text { - High-dose methotrexate with Leucovorine } \\
\text { rescue. } \\
\text { Escalating-dose intravenous methotrexate (no } \\
\text { leucovorinerescue). }\end{array}$ & $\begin{array}{l}\text { - } \\
\text { - } \quad \text {-asparaginase. } \\
\text { High-dose methotrexate } \\
\text { Leucovorine rescue. }\end{array}$ \\
\hline Cranial radiation & No & Yes \\
\hline
\end{tabular}




\subsection{Radiation Therapy}

Radiation therapy is a cancer treatment accomplished through dissipating high-energy $\mathrm{x}$-rays or other types of radiation (example: gamma rays) to damage or destroy leukemia cells. These rays are not sensed or seen by the human eye. Radiation therapy can be external or internal. The first therapy sends energy rays towards the located cancer from a machine outside the body. As for the later, it places a radioactive substance directly into or near the targeted cancer cells through the use of catheters, wires, seeds or needles. The radioactive substance is carefully sealed in its delivery mean. External radiotherapy is used for ALL that has or may spread to CNS and testicles, also for preparing bone marrow for stem cell transplant (PDQ patient's overview, 2016).

\subsection{Hematopoietic Stem Cell Transplant (HSCT)}

HSCT is not the first line option to childhood ALL treatment, yet it is used for treatment of ALL patients who replase. It involves an intravenous (IV) infusion of immature stem cells that have been collected from a donor's bone marrow, peripheral blood, or umbilical cord to reestablish the hematopoietic function in patients. The stem cells donated must be matching to his HLA regardless of whether the donor and recipients are related or not (PDQ patient's overview, 2016).

\subsection{Overall Outcome}

Approximately $98 \%$ attain remission of which $1 \%$ to $3 \%$ die during induction therapy (PDQ patient's overview, 2016) and $85 \%$ are expected to be long-term event-free survivors with over 90\% 5 years' survival (PDQ, 2016).

Despite these good results myelo-suppression and generalized immunosuppression are anticipated consequences of treatment. Toxic effects of therapy can be acute or develops later, which comes in the form of seizures, neoplasms, and neurocognitive impairment. Meningioma's lesions also occur in survivors who receive radiation therapy or not. Other common side effects include anemia, diarrhea, fatigue, fertility issues, hair changes, memory loss, menopause and menopausal symptoms, mouth and throat sores, nausea, taste and smell change, vomiting, weight changes. Whereas osteoporosis, heart problems, vision problems are less common (Owens, 2013; PDQ, 2016).

\section{Health and Nutrition of ALL Children}

\subsection{Nutrition Considerations of ALL Children}

Early detection, enhanced treatment embracing the use of multiple treatment modalities, management of infections and improvement in supportive care has improved the survival rates of pediatrics. These gains in the pediatric oncology treatment have corresponded to an array of unfavorable treatment related effects contributing to increased complexity of assessing acute and long-term nutritional status along with nutritional needs. The onset of these adverse effects maybe acute or delayed (Owens, 2013).

Antioxidant depletion is one of the most commonly described nutrition-related adverse effects of chemotherapy. Other effects include malnutrition, obesity (or increase in body fat), elevated protein turnover seen in treatment of children with high dose of methotrexate and glucocorticoids. Chronic conditions include: endocrine disorders, disorders in growth and pubertal development, hypothyroidism, neurological and neurosensory disorders, cardiovascular disease (CVD), pulmonary disease, osteonecrosis, secondary cancer, fatigue and chronic pain (Owens, 2013).

\subsection{Malnutrion}

Malnutrition is defined generally as an inadequate nutritional state. It may be a subsequent to an imbalance in composition, and utilization of energy, nutrients or both. It may also be a result of cancer or its aggressive treatment and concomitant toxicities. Mucositis and appetite disorders, dysdausia, and xerostoma would alleviate risk of inadequate intake leading to further increased risk of malnutrition (Owens, 2013).

Malnutrition is thought to withdraw appreciable adverse clinical outcomes lessens the quality of life as well as the overall wellbeing. Also of being a risk factor for diminished immunity, altered drug metabolism, leading to drug toxicities, and delayed or prolonged wound healing (Owens, 2013). Regular standard parameters as weight, height, and serum proteins used for assessing nutritional status are often altred in pediatric oncology. Undernutrition can be masked by corticosteroid-induced edema; weight gain due to fluid retention; thereby excluding weight as an accurate nutrition status indicator for a duration of time. Moreover, changes in weight can be related to hydration status during chemotherapy (Owens, 2013).

Serum protein markers are of limited role in identifying malnutrition as there serum concentrations are altered by the liver's synthesis rate, degradation and leakage from the circulatory system. For instance, during inflammation or infection state the liver's acute-phase proteins synthesis as ceruloplasmin, c-reactive (CRP) and ferritin are up-regulated, whereas the synthesis of albumin, retinol binding protein and transferrin (negative acute-phase proteins) are downregulated as a stress response consequence. Lack of conclusive guidelines for assessing or identifying children and young ALL patients who are at risk of malnutrition makes it quite difficult to obtain a precise rate of malnutrition in this population, yet an approximation rate of $46 \%$ was reported (Owens, 2013).

Unrecognized body nutrients depletion (zinc and other micronutrients) as a result of metabolic alterations and other consequences adds further complexity to malnutrition in this population. Leptin hormone is the major regulator of appetite 
and satiety, whereas high levels of this hormone will downregulate appetite and enhance energy utilization. Human studies in cancer patients have shown that there is no sign of elevated levels of leptin during weight loss indicating that the hormone leptin is not involved in the initiation of anorexia in this population (Owens, 2013).

\subsection{Nutritional Assessment and Serum Zinc and Copper Concentrations}

Children with cancer or undergoing chemotherapy with or without radiation are at risk of under nutrition that will eventually end up in one way or another in the reduction of absorbed nutrient intake. However, controversy regarding the influence of cancer treatment on nutritional status exists. Extensive studies concerning the effect of trace elements over the etiology of cancer are conducted in the last few years. Many researchers are attempting to establish a relationship between trace elements (especially zinc and copper) and malignant diseases. For instance, Brown metal studies came to support the association between zinc deficiencies with etiology of cancer. Serum copper levels increases with increased malignant disease activity are being shown by other studies. It has been proposed copper serum might be a good indicator of lymphoma and leukemia's progression as well as a chemotherapy response predictor (Sgarbieri, 2006).

Alongitudinal study was conducted on 45 children with ALL, in order to assess serumzinc and copperduring disease progression. The results have revealed no significant changes in serum zinc levels during treatment, but copper levels have declined with induction phase and then stabilized for the rest of the treatment (Figures 2 and 3) among children with high and low risk groups with a normal serum levels of zinc and copper ranging between $50-120 \mu \mathrm{g} / \mathrm{dl}$ and $70-120 \mu \mathrm{g} / \mathrm{dl}$ respectively (Sgarbieri, 2006).

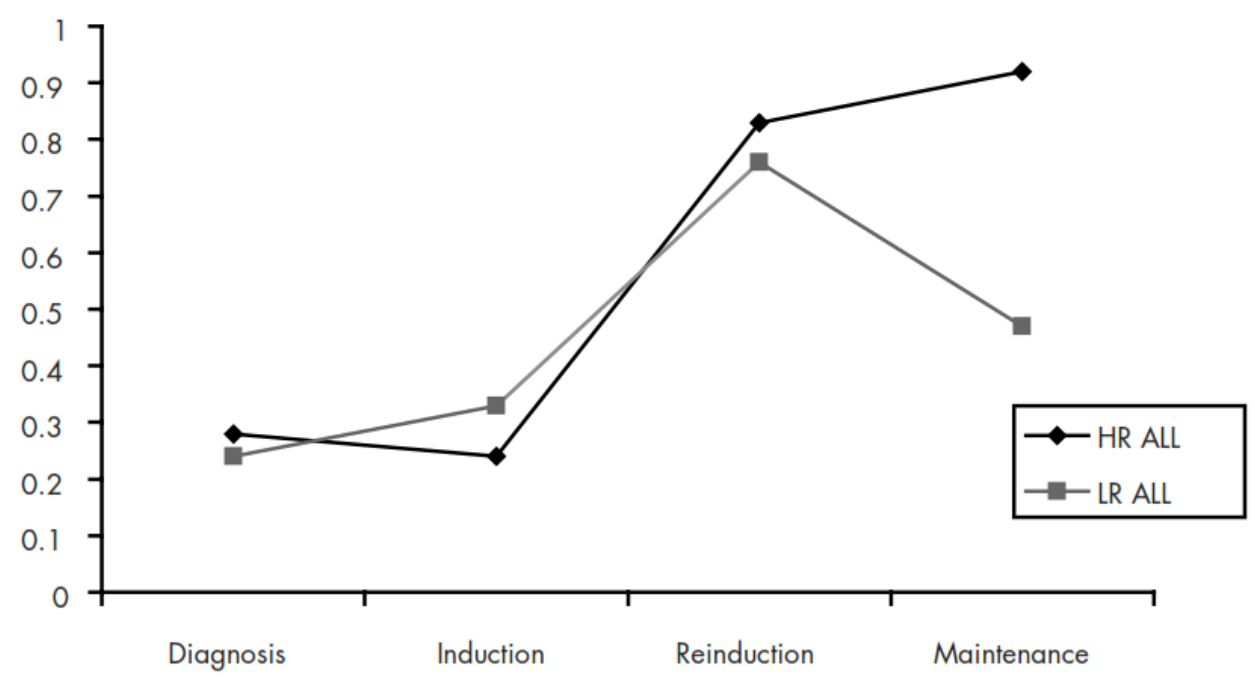

Figure 2. Serum zinc levels of the children with high-risk acute lymphocytic leukemia (HR ALL) and low-risk acute lymphocytic leukemia (LR ALL) at the stages of diagnosis, induction, reinduction and maintenance therapy (Sgarbieri, 2006)

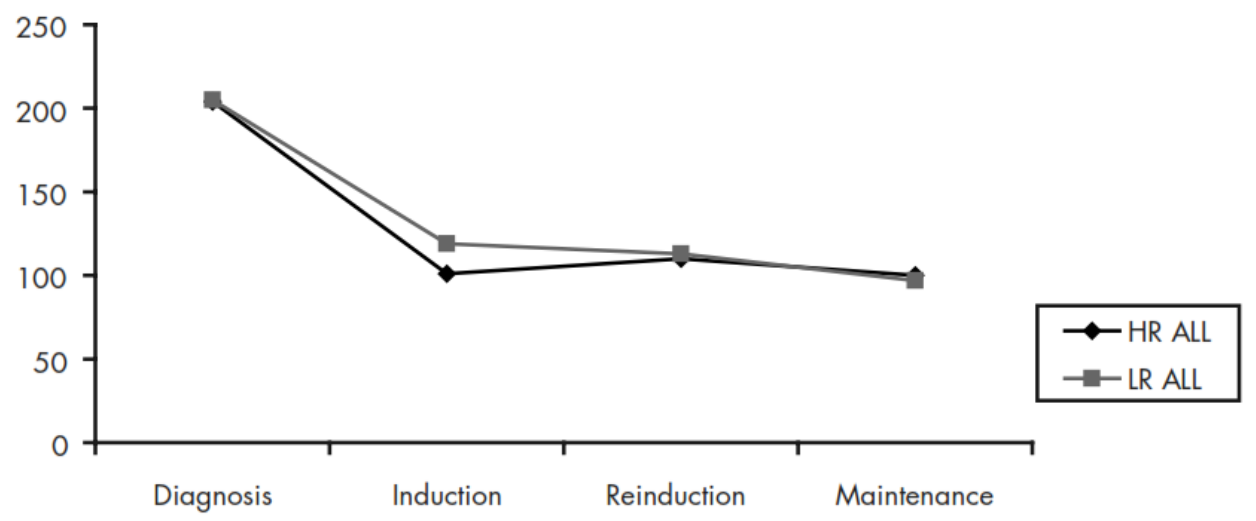

Figure 3. Copper levels in the children with high-risk acute lymphocy tic leukemia(HR ALL) and low-risk acutelymphocytic leukemia(LR ALL) at the stages of diagnosis, induction, reinduction and maintenance therapy (Sgarbieri, 2006) 
According to the figures above serum copper levels were significantly higher at diagnosis compared to normal ranges. Hence many variables such as inflammation and infectious diseases may alter the concentrations of zinc and copper levels, are being investigated to determine their relative contributions. However, no positive correlations were found between these variables and blood concentration. Regardless of the existence of controversy it has been encouraged that blood determination of these two trace elements could be of use in estimating the presence of malignancies, but not as sensitive prognostic factors. A far more extensive and deeper studies concerning basic mechanisms of serum copper and zinc alterations and their significance regarding malignancy is needed (Sgarbieri, 2006).

\subsection{Folate and Vitamin B12 Levels}

B12 deficiency was associated with hematopoiesis disorders and immune response dysfunction inducing an increase in the infection susceptibility. An increase in chemotherapy induced toxicity in rats was demonstrated with folate deficiency and its repletion has been established to alleviate methotrexate toxicity in adults suffering from rheumatological diseases (Tandon, 2015).

Infection and intolerance to chemotherapy were leading causes of children's death in developing countries. An observational study was done in tertiary care teaching hospital in North India. The aim of this study was to thoughtfully shed light on folate and vitamin B12 supplement consideration in deficient children on treatment for ALL in order to decrease short-termmortality. This study enrolled 50 patients of which 21 children have high risk and 29 of low risk ALL. $33(66 \%)$ were shown with undernutrition and about 19 (38\%) and 17 (34\%) had folate and vitamin B12 pre-induction deficiencies, respectively. Four of the children were excluded from the analysis and in the remaining 46, 17 (40\%) suffered from toxic deaths during induction phase, leaving 29 survivers for post-induction (Tandon, 2015). Upon the initial 2 months of chemotherapy serial folate levels examined a persistant and critical decline as shown in figure 4 below (Tandon, 2015).

Folate deficiency was noticed to be a significant determinant of recovery of blood counts and bone marrow at day 14 of induction. Hypoalbuminemia, vitamin B12 and folate deficiencies all were importantly associated with toxic deaths during induction chemotherapy. Moreover, vitamin B12 deficient individuals were verified with dysfunctional immunity (both cellular and humeral), low CD4 and CD8 counts, lessened natural killer (NK) cells function and low immunoglobin levels. This might explain the elevated toxic death hazards noticed in vitamin B12 deficient children being observed (Table 2) (Tandon, 2015).

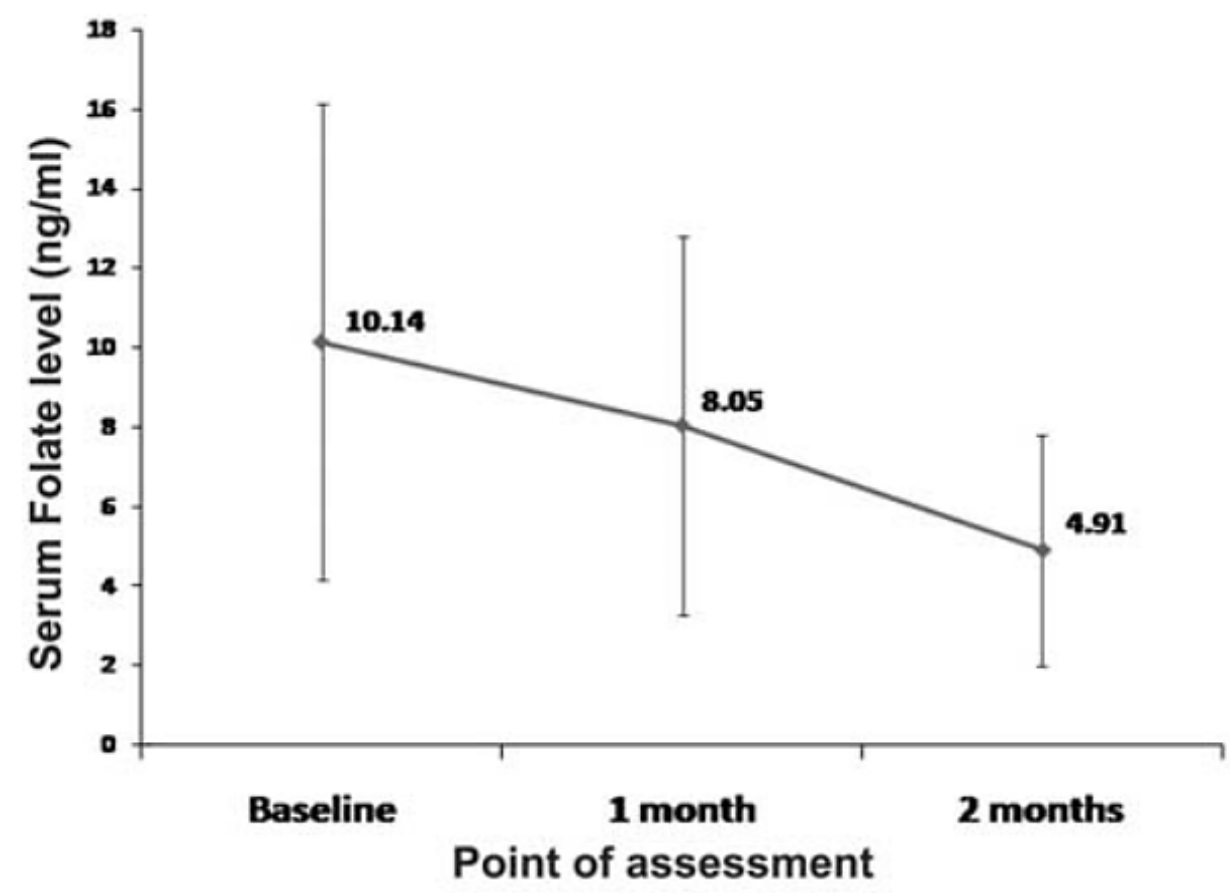

Figure 4. Trend of serum folate levels in patients with acute lymphoblastic leukemia on chemotherpy (n=29) (Tandon, 2015) 
Table 2. Effects of Pre-treatment Vitamin B12 and Folic Acid on Complications in Children on Chemotherapy for ALL ( $\mathrm{N}=46)$ (Tandon, 2015).

\begin{tabular}{lcccc}
\hline Variable & No. & $\begin{array}{c}\text { Bone marrow recovery } \\
\text { Incomplete } \\
(n=33)\end{array}$ & $\begin{array}{c}\text { Delayed } \\
(n=27)\end{array}$ & $\begin{array}{c}\text { Toxic } \\
\text { induction } \\
\text { deaths } \\
(n=17)\end{array}$ \\
\hline $\begin{array}{c}\text { Serum folate } \\
\quad<4 \mathrm{pg} / \mathrm{mL}\end{array}$ & 19 & 18 & 17 & 10 \\
$\quad \geq 4 \mathrm{pg} / \mathrm{mL}$ & 27 & 15 & 10 & 7 \\
$\begin{array}{c}\text { Vitamin B12 } \\
\quad<203 \mathrm{ng} / \mathrm{mL}\end{array}$ & 17 & 10 & 10 & 12 \\
$\geq 203 \mathrm{ng} / \mathrm{mL}$ & 29 & 23 & 17 & 5 \\
$\begin{array}{c}\text { Serum albumin } \\
<3.5 \mathrm{mg} / \mathrm{dL}\end{array}$ & 15 & 8 & 7 & 9 \\
$\geq 3.5 \mathrm{mg} / \mathrm{dL}$ & 31 & 25 & 20 & 8 \\
$\begin{array}{c}\text { WAZ-score } \\
<-2\end{array}$ & 33 & 23 & 20 & 13 \\
$\geq-2$ & 13 & 10 & 7 & 4 \\
\hline
\end{tabular}

Table 2 shows common outcomes associated with folate deficiency as incomplete bone marrow recovery at day 14 , delayed peripheral blood counts recovery and toxic induction deaths during induction phase compared to folate replete. As for toxict induction deaths were more common in vitamin B12 deficient children in contrast to vitamin B12 loading. However, United States (US) study has stated that folate levels are elevated following the induction of ALL children and falls following consolidation with higher methotrexate doses. The fall in folate level is due to methotrexate alone, and possibly much higher during the preceding treatment phases along with higher doses of methotrexate and thus a higher toxicity consequence. Supplementing folate and vitamin B12 may offer the solution to avoid toxicities in deficient patients. This is more relevant in patients who have a predominant vegetarian diet, obligatory diet restrictions during treatment, and concomitant antifolate therapy. Meanwhile controversies are being loaded against folate supplement during cancer therapy due to the concern that folate may induce limits to the efficacy of chemotherapy and supports neoplastic cell growth (Tendon, 2015).

\subsection{Mucositis}

A significant number of children developed mucositis, a debilitating complication of cancer treatment, particularly chemotherapy and radiotherapy. Mucositis can lead to several problems including pain, oral hemorrhage, nutritional problems (risk of malnutrition) as a result of inability to eat and increase risk of infection due to open sores in the mucosa layer. It has a major effect on the patient's quality of life and can be dose limiting (requiring a reduction in subsequent chemotherapy doses). Regular oral care is superior to treatment. Palifermin (Kepivance) medication was approved by the US Food and Drug Administration (FDA) to prevent or treat severe oral mucositis in adults with hematologic malignancies and undergoing high chemotherapeutic doses (with or without radiation) proceeded by HCST (Owens, 2013).

\subsection{Sinusoidal Obstructive Syndrome}

Also known as Hepatic Veno-occlusive disease occurs after HSCT and in some types of chemotherapies. It is a result of endothelial cell injury in the blood vessels which flows within the liver and surrounding organs that are supported by Portal circulation leading to their blockage. This is induced by increased oxidative stress. It is believed that antioxidant glutathione (GSH) deficiency plays an ample role in the pathogenesis of this complication. Reducing the frequency of such complication can be managed through a diet rich in antioxidants (AOX) and adequate protein and energy (Owens, 2013).

\subsection{Nutrient Drug Interaction}

Food and nutrients can also affect the rate at which human body processes or eliminates medicine. For example, methotrexate inhibits folate metabolism. Homeostasis of potassium and magnesium are altered by Cyclosporine necessitating the nutritional replenishment due to their serum depletion. Moreover, long term use of gluco-corticosteroids abet hyperglycemia, fluid retention, weight gain (fat mass) resulting in altered body composition, electrolyte abnormalities, and increased needs of calcium, zinc, vitamin $\mathrm{C}$ and D (Owens, 2013).

A considerable increase in obesity are reported in leukemic patients prescribed gluco-corticosteroids in conjunction with methotrexate, and more precisely in female ALL patients treated with cranial radiation. Childhood survivors are presented with body mass index (BMI) $\geq$ $25 \mathrm{~kg} / \mathrm{m}^{2}$ (Owens, 2013).

\subsection{Oxidative Stress}

Depletion of antioxidants (AOX) is a common nutrition related adverse effect that is mostly problematic during conditioning phase. This puts the body under oxidative stress; body's imbalance between pro-oxidants and antioxidants as part of some chemotherapeutic drugs technique to destroy cancer cells. As many of these chemotherapeutic drugs produce free radicals as oxygen $\left(\mathrm{O}_{2}\right)$ free radicals which induce depletion of the body's AOX stores while attempting to neutralize them. During intense conditioning phase the patient receives drug doses with limited time interval between them, placing the patient into remission from their cancer. This would leave the patient quite ill and have lessened tolerant ability to resume an adequate nutritional intake in perusing AOX replenishment from diet (Owens, 2013). 


\section{Supplementing ALL Pediatric}

\subsection{Dietary Supplements in Cancer}

Complementary and Alternative Medicine (CAM) are not held as part of the conventional medicine, due to some part of its mysteriousness and to its expanding constituent fact. CAM consists of vast array of dissimilar therapies. It is commonly used by the general public, an approximate between $35 \%$ and $50 \%$ of U.S children with cancer take dietary supplements, and $6 \%$ to $91 \%$ use CAM. The most commonly reoccurring reasons for consuming dietary supplements by individuals with cancer are to assist coping with adverse side effects of conventional treatment, to strengthen conventional anticancer therapy, and to prohibit secondary malignancies. A dietary supplement for example may be composed of a single or multiple dietary ingredients that append vitamins, minerals, herbs, amino acids, and other botanicals that are administered orally in the form of capsules, liquids, pills or tablets. Oral pain and trouble swallowing are as sociate unfriendly impacts of mucositis, thereby increasing the risk of dehydration and/or malnutrition (Owens, 2013).

A few supplements have been investigated in regards to their capability to modulate mucositis progression; Traummel $S^{\mathrm{R}}$ is one such product. It's a homeopathic cure formed of highly diluted botanical extracts; a drug made from parts of plan, roots, leaves, bark and berries; and minerals . According to a randomized controlled double-blinded clinical trial where 32 pediatric patients have been enrolled at multi-institutional sites showed its effectiveness in reducing stomatitis and mucositis (Table 3). A contradicting result of anotherdouble-blinded randomized study involving 181 patients, having a wider age spectrum, who are undergoing HSCT in a subsequent international multi-center didn't confirm the effectiveness of Traummel $\mathrm{S}^{\mathrm{R}}$ in mucositis treatment. This later contemplate acquired a pattern of diminished opiate use to mouth torment with Traummel $S^{R}$ usage. Glutamine (GLN) is another supplement used to prohibit or lessen the severity and/or the duration of mucositis in children undergoing HSCT. GLN has multiple roles in the human body and acts as a precursor of glutamate, which is required for the formation of major AOXGHS. A single possible step back to GLN use is that it necessitates the use of substantial amount of fluid administration that would contraindicate it for children who are undergoing HSCT which requires fluid restriction (Owens, 2013).

Table 3. Overview of nut rition-related pediatric oncology studies - mucositis and nutrition care practices (Owens, 2013).

\begin{tabular}{|c|c|c|}
\hline Author & O verview & Results \\
\hline $\begin{array}{l}\text { Sencer, S.F. et } \\
\quad \text { al. } 2012\end{array}$ & $\begin{array}{l}\text { International multi-institutional, double-blinded, randomized trial. } \\
\text { A total of } 181 \text { participants bet ween the ages of } 3-25 \text { years; } \\
\text { evaluation of the effectiveness of Traumeel S® in the treatment of } \\
\text { mucositis. }\end{array}$ & $\begin{array}{l}\text { Lack of confirmation of Traumeel } \mathrm{S} \AA \text { : } \\
\text { - } \quad \text { As an effective treatment for CT-induced } \\
\text { mucositis. } \\
\text { - } \quad \text { Found trend toward reduced narcotic used. }\end{array}$ \\
\hline $\begin{array}{l}\text { Ladas, E.J. et } \\
\text { al.2006 }\end{array}$ & $\begin{array}{l}\text { A total of } 223 \text { COGinstitutions were invited to complete a survey } \\
\text { as a means to ascertain the degree of consensus practices utilized } \\
\text { in assessment of nutritional stat us; either a MD, RD or RN } \\
\text { completed the survey. }\end{array}$ & $\begin{array}{l}\text { A total of } 120 \text { COG facilities responded and it was } \\
\text { found that nutritional assessments were performed } \\
\text { using numerous different indices to: } \\
\text { - Identify nutritional status. } \\
\text { - } \quad \text { Classify malnutrition and its severity. }\end{array}$ \\
\hline $\begin{array}{c}\text { Thornley, I. et } \\
\text { al. } 2004\end{array}$ & $\begin{array}{l}\text { Pilot Study, } 37 \text { participants Assessment of the benefit of the AOX: } \\
\text { ursodexycholic acid, vitamin E, and folinic acid in children } \\
\text { receiving PN undergoing HSCT. }\end{array}$ & $\begin{array}{l}\text { The most distinct benefits were found in high risk } \\
\text { patients assessed by: } \\
\text { - Shorter time period to engraftment. } \\
\text { - Reduced severity and incidence of } \\
\text { mucositis. } \\
\text { - Decreased severity of liver toxicity. }\end{array}$ \\
\hline $\begin{array}{c}\text { Oberbaum, } M . \\
\text { et al. } 2001\end{array}$ & $\begin{array}{l}\text { A randomized, controlled clinical trial, } 32 \text { participants, ages } 3-25 \\
\text { years, evaluation of TRAUMMEL S® in the treatment of } \\
\text { mucositis. } \\
\text { WHO grading score used to assess the severity of mucositis. }\end{array}$ & $\begin{array}{l}\text { TRAUMEEL S® may have significantly lowered: } \\
\text { - } \quad \text { Severity and duration of oral mucositis } \\
\text { induced by SCT. } \\
\text { - } \quad \text { Mean mucositis days for placebo group } \\
24.3 ; 10.4 \text { for treatment group. }\end{array}$ \\
\hline
\end{tabular}

®HEEL Corporation SCT/HSCT: stem cell transplantation/hematopoietic stem cell transplant; CT: chemotherapy; ALL: acute lymphoblastic leukemia; AML: acute myeloid leukemia; PN: parenteral nutrition; WHO: W orld Health Organization. 


\subsection{Antioxidant Supplements in Cancer}

Several cancer therapies induce oxidative stress as a part of their mode of action to cause cancer cells destruction. Depleted or reduced AOX levels have been obtained in patients at the conditioning phase, especially vitamin C, selenium and vitamin E. This has promoted controversy concerning the supplemental benefit or harm of AOX at levels well beyond the recommended daily intake during cancer treatment. These therapies promote the production of ROS (free radicals) as part of their anticancer impact. Anthracyclines, arsenic trioxide, cytarabine, and vincristine are USFDA approved anticancer treatments which generate ROS (Owens, 2013).

The fear of AOX could impair the action of certain types of anticancer drugs hasn't been confirmed by clinical trials, yet not all effects of anticancer therapies include the generation of ROS. Thereby considering the potential benefit of AOX supplementation to enhance the nutritional status and reduce chemotherapy related toxicities in some patients, although inconsistency in study results exists. ROS possesses both a positive and negative function in the proliferation and survival of the cell. Normally the body maintains equilibriu m between ROS production AOX activity. The loss of this balance is important in leukemia cells, which contains moderately elevated ROS levels as a result of changes in prooxidant and AOX pathways. These pathways contribute to the susceptibility to cancer treatment. Once ROS levels overwhelm the manageable cell's capacity, oxidative damage befalls on lipid peroxidation and deoxyribonucleic acid (DNA) damage. Withholding higher levels of ROS happens in all four forms of leukemia. Intermittently leukemic cells are deregulated in both expression and function of diverse AOX pathways, thus playing a significant role in the survival, growth, and progression and resistance therapy. Plus raised ROS levels promote leukemogenesis even with the contempt from traditional cancer treatment. On the other hand the oxidant balance in leukemia favors ROS production that produces complexes having the abeyant of causing direct destruction to cancer cells or promoting growth and survival inhibition of potential cancer cells (significant for clinical application). Altogether, leukemic cells down-regulate AOX and by that mean stimulating ROS signaling and genomic instability. However, upon remedy with ROS-producing agents, many AOX enzymes that have the opportunity to be up-regulated and the probability to induce anticancer treatment resistance. Improved understanding from claiming oxidative party on leukemia will prompt further headway in the medicine for leukemia and decrease in the co-partnered toxicities prompt confirmation based dietary rules for the utilization or non-use of AOX supplementation throughout and then afterward anticancer therapy for pediatric leukemia. Table 4 below shows an overview of nutritional related pediatric oncology studies in association with antioxidants status and or use (Owens, 2013).

Table 4. Overview of nut rition related pediatric oncology studies — antioxidants (Owens, 2013).

\begin{tabular}{|c|c|c|}
\hline Author & Overview & Results \\
\hline $\begin{array}{c}\text { Radhakrishnan, N. et } \\
\text { al. } 2012\end{array}$ & $\begin{array}{l}\text { Case control study, } 45 \text { newly diagnosed children } \\
\text { with ALL serum fasting levels of zinc, selenium, } \\
\text { retinol and tocopherol were compared to an age- } \\
\text { matched control group of } 20 \text {. }\end{array}$ & $\begin{array}{l}\text { Patients with lower serum levels of selenium and } \\
\text { tocopherol at diagnosis were found to be at greater risk } \\
\text { for: } \\
\text { - } \quad \text { Febrile neutropenia. } \\
\text { - } \quad \text { Sepsis during the first } 8 \text { weeks of therapy. }\end{array}$ \\
\hline $\begin{array}{l}\text { Papageorgiou, M. et } \\
\quad \text { al. } 2005\end{array}$ & $\begin{array}{l}\text { Observational st udy was } 80 \text { patients receiving CT. } \\
\text { T AC and cT AC levels were evaluated. }\end{array}$ & $\begin{array}{l}\text { During CT: } \\
\text { - } \quad \text { TAC and cTAC levels progressively declined. } \\
\text { - } \quad \text { TAC and cTAC levels remained low for } 6 \text { months } \\
\text { post treatment. } \\
\end{array}$ \\
\hline $\begin{array}{l}\text { Aquino, V.M. et al. } \\
2005\end{array}$ & $\begin{array}{l}\text { Double-blinded randomized placebo-controlled } \\
\text { study. } 120 \text { children T wenty-eight days following } \\
\text { HSCT or hospital discharge in which } 50 \% \text { received } \\
\text { oral-glycine and } 50 \% \text { received oral-GLN. }\end{array}$ & $\begin{array}{l}\text { - The GLN group was found to havea reduction in } \\
\text { the number of days requiring narcotics for } \\
\text { mucositis. } \\
\text { GLN appears to be both safe and effective in } \\
\text { decreasing the severity ofmucositis. }\end{array}$ \\
\hline Kennedy, E. et al. 2004 & $\begin{array}{l}\text { Multi-centered, prospective, observational study of } \\
103 \text { children diagnosed with ALL. The AOX levels } \\
\text { of vitamin A, E, ascorbate, } \beta \text {-carotene, total } \\
\text { carotenoid was evaluated. Dietary intake of AOX: } \\
\text { calculated based on } 24 \mathrm{~h} \text { Food recall and food } \\
\text { frequency questionnaire obtained at three separate } \\
\text { intervals. }\end{array}$ & $\begin{array}{l}\text { - } \\
\text { - } \\
\text { Higher dietary intake of vitamin } \mathrm{E} \text { at } 3 \text { months } \\
\text { correlated with a lower infection rate. } \\
\text { Higher dietary intake of vitamin } \mathrm{C} \text { and } \beta \text {-carotene } \\
\text { at } 6 \text { months correlated with decreased toxicity } \\
\text { related to therapy. }\end{array}$ \\
\hline
\end{tabular}

ALL: acute lymphocytic leukemia; MDA: malondialdehyde; T AC: total AOX capacity; CT: chemotherapy; TUNEL: terminal deoxynucleot idyl transferase dUTP nick end labeling; AOX: antioxidants; GLN: glutamine; cTAC: corrected total; AOX capacity; NAC: N-acetylcysteine; Glu.Px: glut athione peroxidase; TNF- $\alpha$ : tumor necrosis factor alpha; HSCT: hematopoietic stem cell transplantation. 


\subsection{Glutamine Enriched Nutrition Therapy in ALL Pediatrics}

Catabolism predominates in children with ALL. They have a major caloric and protein intake shortage, and raised body protein consumption, meanwhile liver infiltration and damage may extend its impact on protein synthesis. Implementing L-ASP-containing chemotherapy exerts a further inhibition to protein manufacturing, leading at the end to protein malnutrition, hypersensitivity, blood clotting abnormalities, gastrointestinal symptoms, lowered immune response, increased mortality, and impacting the following chemotherapy. Owens declares that reduced nutritional status of children with leukemia after chemotherapy is hazardous, for it can reduce immune function, alter medicine metabolism, improve medicine toxicity, and extend wound healing. The American Society of Parenteral and Enteral Nutrition (ASPEN) published clinical nutritional guidelines for cancer patients, emphasizing on the lack of proof that shows nutrition therapy can induce tumor cells growth. It also supplied ALL children with a theoretical ground base to put nutrition therapy in to action during chemotherapy (Han, 2016).

GLN is the most abundant non-essentialamino acid in the muscle, estimating for $60 \%$ of total free amino acids in the human body. The multifaceted roles of GLN were evident, for example Gaurav $\mathrm{K}$ et al. discovered its mucosal injury and gastrointestinal toxicity reducing effects that are instigated by radiotherapy and chemotherapy, and 5-FU (fluorouracil drug) respectively. On top of that, it reduces the effect of paclitaxel-related myalgia and arthralgia, prohibiting paclitaxel neurotoxicity, relieve the immunecompromised state of laboratory animals after MTX (methotrexate) chemotherapy, and stimulate the recovery of neutrophils in patients with myeloid leukemia after chemotherapy. However, the GLN mechanism is not entirely understood. Clinical researching has been conducted to uncover the mechanisms behind GLN mode of action, including that of Goto discovered in the study of acute myeloid leukemia (AML) that GLN might take a significant part in energy metabolism in the Krebs cycle through its influence on redox balancing. Emadi A et al. realized that the selective inhibition effects of GLN came to growth inhibition of myeloid leukemia cells with defects in the IDH gene. Nonetheless few main numbers of reports considered GLN as an immune and nutrition agent and respectively utilized its effect on both the immune function and nutrition status in children with ALL. One study in china aimed at investigating the impact of GLN-enriched nutrition therapy that was applied through chemotherapy on the nutritional status and immune function, by means of exploring the mode of nutrition therapy for childhood leukemia, help expand the youth tolerance to chemotherapy, augment immunity, improve their quality of life, and even aid in long-term prognosis advances. Assessment for dietary state indicators over leukemic kids with leukemia after chemotherapy is quite complicated. The standard parameters used to evaluate those dietary statuses for children with leukemia might change precise often. For instance, edema can mask malnutrition after steroid therapy hence impairing use of weight as an accurate nutrition status indicator. Although edema was assessed in the study were $50 \%$ the controlled group (received peptamen) showed signs of edema of which 4 cases were severe (Table 5), meanwhile 20.83\% were detected in the treatment group (received peptamen + glutamine) of which 1 case was severe after 4 weeks of therapy (Han, 2016).

Serum protein markers can show bias when used for assessing malnutrition affected by infection and also sepsis, thus retinol binding protein is the most sensitive indicator. CHI (creatinine-height index), albumin, prealbumin, urinary hydroxyproline index were compared between the two groups. At the end of the treatment (4 weeks), the percentages of $\mathrm{CD} 3+, \mathrm{CD} 4+, \mathrm{CD} 4+/ \mathrm{CD} 8+$, NK cell were significantly decreased $(\mathrm{P}<0.01)$ in the two groups (Table 6$)$. This implies that the cellular immune in both groups was utterly damaged; proposing that strengthened glutamine therapy can promote the recovery of immune function in ALL patients. This contemplate found that GLN-enriched treatment (dose of $0.4 \mathrm{~g} / \mathrm{kg} . \mathrm{d}$ ) might altogether enhance those dietary status about kids with ALL, what's more it could additionally enhance the body's digestion system and nitrogen balance, push toward protein synthesis, and expand the downright amount of lymphocytes. Concerning the safety of glutamine submission, it was established that a GLN dose of 3832$4515 \mathrm{mg} / \mathrm{kg}$ for 13 weeks presented no toxicity reactions in rats in a chronic toxicity test by Wong (Han, 2016).

Every single patient throughout treatment didn't show unfriendly reactions, including hypersensitive reactions, diarrhea, bloating, vomiting, and constipation, and most patients demonstrated a handy tolerance (Han, 2016).

Table 5. Edema observed after 4 weeks' treatment (Han, 2016).

\begin{tabular}{|c|c|c|c|c|}
\hline Parameters & $\mathbf{n}$ & Cases of edema & Cases of severe edema & Percentage of edema $(\%)$ \\
\hline Treatment group 24 & 24 & 5 & 1 & $20.83 \%$ \\
\hline Control group & 24 & 12 & 4 & $50 \%$ \\
\hline
\end{tabular}


Table 6. The differences of cellular immune function in the two groups before and after treatment (Han, 2016).

\begin{tabular}{|c|c|c|}
\hline Parameters & $\begin{array}{c}\text { Control group }(\mathrm{n}=24) \\
\text { Before chemotherapy after4 weeks }\end{array}$ & $\begin{array}{c}\text { Treatment group }(\mathrm{n}=24) \\
\text { Before chemotherapy after4 weeks }\end{array}$ \\
\hline CD3+ & $69.23 \pm 1.1555 .20 \pm 2.96 \mathrm{a}$ & $70.55 \pm 3.05 \mathrm{a} 63.67 \pm 1.64 \mathrm{~b}$ \\
\hline $\mathrm{CD} 4+$ & $32.74 \pm 2.8526 .30 \pm 2.70 \mathrm{a}$ & $33.94 \pm 2.7533 .83 \pm 1.56 \mathrm{~b}$ \\
\hline $\mathrm{CD} 8+$ & $28.45 \pm 0.9828 .00 \pm 0.88$ & $27.79 \pm 0.89 \mathrm{a} 26.50 \pm 1.02 \mathrm{~b}$ \\
\hline $\mathrm{CD} 4+/ \mathrm{CD} 8+$ & $1.16 \pm 0.580 .93 \pm 0.10 \mathrm{a}$ & $1.22 \pm 0.651 .28 \pm 0.84 \mathrm{~b}$ \\
\hline NK cell \% & $23.95 \pm 2.3416 .23 \pm 4.15 \mathrm{a}$ & $25.47 \pm 2.01 \mathrm{a} 19.54 \pm 4.06 \mathrm{~b}$ \\
\hline
\end{tabular}

a) compared with before chemotherapy, $\mathrm{P}<0.05$; b) compared with control group, $\mathrm{P}<0.05$.

\section{General Nutrition Recommendations for ALL Pediatrics during and after Therapy}

\subsection{Weight Management throughout Treatment and Thereafter}

Weight control is universally important for the overall wellbeing making weight reduction for overweight and obese ALL patients and survivors a rational goal for intervention. During treatment receiving children experience substantial weight gain and again during maintenance phase, regardless of whether the child is receiving radiation or not as well for the age, gender, weight status at diagnosis. Furthermore, patients who are below average BMI (body mass index) appeared to experience a greater degree of weight gain during and after treatment equated to those associated with normal or high BMI at diagnosis. This may reflect a greater impact of cancer therapy on catch-up growth for leaner individuals at diagnosis. Additionally, some therapy agents may contribute to weight gaining and thus resulting in obesity such as corticosteroids. Steroids are known to be critically elaborated in energy intake, storage and metabolism, by which prolonged consumption may increase energy intake and percentage of fat mass. Therefore, some doctors and physicians considered to follow strategies that reduce the input of steroids in the maintenance phase in order to meliorate some of the unhealthy weight gained during this period. Other chemotherapeutic agents as anthracyclines and vincristine are also involved in weight gain by impairing cardiovascular fitness and muscle strength, and subsequently reducing physical activity level (Zhang, 2016). Therapy impact the hypothalamic- pituitary region in a developing brain by impairing regulations and causing food craving. An assessment for some survivors established that craving for fast foods was the most relevant followed by sweets, carbohydrates, fat in order. However, craving doesn't explain the obesity risk. Relying of the weight status as an indicator of long term energy balance, the majority of long-term survivors consumed excess energy than what they expend contributing to risk of overweight and obesity. Childhood survivors usually meet their protein intake within dietary requirements and the carbohydrates are primarily met by low fiber highly processed foods, and fats are consumed above the dietary reference intake (DRI's) (Tylavsky, 2013). On top of that, an energy gap of 500kcal/day was estimated between energy requirements with recommended physical activity and energy expenditure, thus contributing to risk of obesity. Worth to note that child's growth accounts for a total of 1-2\% of the energy expenditure. Causes of obesity are speculative which implicates the need of knowledge in preventing and treating obesity in vulnerable targets, however we can to a certain degree link it to reduced energy expenditure, increased energy intake, reduced physical activity. This later reason is due to sedentary life style and loss of FFM (fat free mass). Thus, promoting an average of $60 \mathrm{mn} /$ day moderateto-vagarious activity level such as brisk walking and swimming ( $250-400 \mathrm{kcal} /$ day $)$ presenting an advantage to reduce fat mass and increasing FFM (Zhang, 2016). Recent evidence designated that children are having difficulty reversing unhealthy eating habits and sedentary behaviors after treatment completion (Zhang, 2016). Physical alone may not adequate to increase the total energy expenditure by an average of $500 \mathrm{kcal} /$ day hence a reduction in the energy intake is also desired to achieve energy balance in childhood cancer survivors. Obesity from the other side augments potential risk to previously raised chronic conditions in childhood cancer survivors and impacts the mortality and morbidity (Zhang, 2016).

\subsection{Caution to Hypertriglyceridemi a during the Treatment of Pediatric ALL}

The administration of asparaginase and steroids causes significant alteration in serum lipid levels specifically that of triglyceride serum levels. Where corticosteroids surge triglyceride synthesis and increase activity of lipoprotein lipase (LPL), a key enzyme required for the hydrolysis of triglycerides. Even though asparaginase might inhibit the activity of LPL, but when combined with steroids triglyceride-rich lipoproteins are rapidly formed but insufficiently cleared from the system. Levels of triglyceride above $1000 \mathrm{mg} / \mathrm{dl}$ are not associated with pancreatitis and osteonecrosis in contrast to general population, were this level may elevate risk of pancreatitis and induce hyperviscosity syndrome, and may lead to thromboembolic events and osteonecrosis. Meanwhile, children have 2.5 to 3 times higher risk of thrombosis during treatment. Cases of triglyceride levels as $20600 \mathrm{mg} / \mathrm{dl}$ (normal $<130 \mathrm{mg} / \mathrm{dl}$ ) and cholesterol levels rose as $1640 \mathrm{mg} / \mathrm{dl}$ (normal<200mg/dl) were reported through therapy. Hypertriglyceridemia 
incidence is under-diagnosed but transient and generally benign. Symptoms of hypertriglyceridemia episodes include diarrhea, abdominal pain, fatigue, nausea and vomiting, sometimes blurred vision. Therefore, management with dietary modifications and monitoring without altering chemotherapy is required but the pharmacotherapy (lipid lowering drugs) administration to reduce the symptoms and complications in children during treatment requires further investigations (Bhojwani, 2015).

\subsection{Bone Health and Status}

Cancer survivors are vulnerable to the exaggeration of the aging process exclusively reduced bone mineral density (BMI). A complete 110-item food frequency questionnaire displaying the dietary intake over previous year presenting that less than $30 \%$ of the participants met the recommended dietary intakes for vitamin $\mathrm{D}$, calcium, potassium, magnesium, regardless of age. The energy intake from sweets were $70 \%$ higher than recommended and they are less probably to have a healthy body weight (participants $>19$ years old are more likely to be overweight). A suboptimal ingestion of minerals combined with insults from ALL therapy to bone metabolis $m$ in addition to possible coexisting endocrinopathies would predispose to a greater jeopardy of osteoporosis and other chronic diseases. Vitamins are directly or indirectly affect the bone metabolism through endocrine and/or paracrine system by modulating calcium homeostasis (Tylavsky, 2013). Some studies have linked between vitamin D deficiency and some cancer development and progression. Moreover, epidemiologic studies confirmed that active vitamin D metabolite $(1, \quad 25$ Dihydroxycholecalciferol) may arrest cell cycle in vitro, and in vivo experiment suggests that vitamin $\mathrm{D}$ deficiency accelerates development of autoimmune disease and cancer (White, 2016). Vitamins A, C, E, and K are linked to bone metabolism via collagen synthesis or bone turnover, and BMD and fractures in adults whereas their importance is only now being examined in children. Fruits and vegetables are excellent sources of potassium and besides they provide bicarbonates, essential oils and monoterpenes which may be beneficial to bone metabolism (Tylavsky, 2013).

\subsection{Effect of Garlic Compounds on Leukemic Cells}

The majority of drugs used in the induction therapy are nonselective for malignant cells. Oral garlic was evident to be effective in reducing the occurrence of esophageal and gastric cancer. T-helper-type 1 (Th1) responses are significant in immune response against malignant cells, viruses and microbial infections. T-cell immune function has been shown to be compromised in children with ALL before and during treatment, in addition to impaired production of T-cell IFNy (interferon) and IL-2 (interleukin 2). TNF $\alpha$ (tumor necrosis factor) is shown to inhibit tumor cell growth in a variety of hematological malignancy and stimulate immune response to tumors. Drugs may further impair antleukemic response, in contrast to effect of garlic compounds which didn't impair T-cell proliferation or Th1 responses in children with ALL or in a normal healthy population. Ajoene, a garlic-derived compound has been shown to inhibit proliferation and promote apoptosis in human leukemia cells, and induce apoptosis in myoblasts. Allitridium, another garlic-derived compound was being used intravenously over 3 years in China to successfully treat Cryptococcus neoformans and other fungal infections (Davis, 2003). ALL patients are susceptible to a variety of bacterial, fungal and viral infections, but garlic compounds presented to own antimicrobial properties in preventing and treating common infective organisms in this population as Escherichia coli. Intravenous garlic compounds are shown to be safe and effective in the treatment of human invasive fungal infection (anti-fungal activity). Thus garlic compounds have proven to stimulate the immune effector cells and modulate inflammatory cytokine production; a potential therapeutic benefit in ALL treatment (Hodge, 2008).

\subsection{Sulforaphane Induces Cell Arrest and Apoptosis in ALL Cells}

Kale, Brussel sprouts, Collard green, Broccoli, Cabbage, Mustard greens, Cauliflower and Turnip are food sources contacting sulforaphanes (SF). It is a group of phytochemicals found in cruciferous plants known as isothiocyanates, helps in preventing the development of cancer. Mainly through the inhibition of certain body enzymes from activating cancer-causing agents and stimulating body to produce other enzymes that clean carcinogens out of the system before they damage the cells. This chemopreventive property is recognized to the activity of isothiocyanates derived from the metabolism of glucosinolates; that accumulates in cruciferous vegetables; through the conversion of glucosinolate glucoraphanin to SF by the action of myrosinase enzyme that is present in the vegetable and gut bacteria. SF is highly reactive and hydrophobic compound that can modify cellular function by entering to it and conjugating to glutathione and secreted via mercapturic pathway, or it forms thionacyl adducts with the thiol groups of not yet recognized proteins in cancer cells. Epidemiologic studies directed in US establish that individuals who consume a diet rich in cruciferous vegetables had lower occurrence of breast, lung, prostate and colon cancer. Moreover, SF prompts apoptosis in several cancer cell lines and has been tested in animal models of human cancer, as T-cell leukemia, breast, colon and prostate cancer by targeting Caspases, PARP, p21, p53 and Bax. SF also causes G1/S and G2/M cell cycle arrest via altering levels of cyclin A, cyclin B1, cyclinD1, P21, and tumor suppressor KLF4. Recent studies show that SF restricts cancer cell survival through inhibiting PI3K/AKT signaling of prostate cancer. Therefore, SF dose-dependent treatment has confirmed its preventive and therapeutic function in solid 
tumors and might have the potential implication in therapeutic use (Suppipat, 2012).

\subsection{Nutrition Recommendations to Be Considered during Cancer Treatment}

1) Careful food handling: caution is given from the beginning if the preparation process of food till the time of delivery to ALL children during treatment. The food has to be clean, hot and sealed to avoid risk of microbial infection. No leftover is allowed to be consumed by the child under any circums tances.

2) Avoiding sugars: no sugary foods/sweets are allowed to be given, since sugar is the main fuel for cancer cells. This will put down the child's immune system(Eldridgre, 2004).

3) Focusing on immune reinforcing foods: Consume foods that strengthen the immune system as garlic, onions, cruciferous vegetables and curcumin due to their immune boosting effect (Suppipat, 2012; Hodge, 2008).

4) Following restricted diet: even though diet is nutrient dense, but its low in carbohydrates and fat (minimum DRI recommendation), but this may result in increased child craving to sweets and fast foods According to the dose of steroids being administered to the child we restrict salt, sugar and fat. A dose of less that $10 \mathrm{mg}$ we reduce salt intake, if the dose is increased up to $20 \mathrm{mg}$ salt is totally restricted from the diet, if the dose is between $20-60 \mathrm{mg}$ then no salt no simple sugar is allowed and finally a dose above $60 \mathrm{mg}$ implies to no salt, no sugar, low fat diet (reduction in saturated and trans fat intake) (Eldridgre, 2004).

5) Considering other parameters: attention is paid to the child's age, gender, weight and height at diagnosis in order to estimate their total energy requirements and considering further adjustment according to the case of treatment (PDQ, 2016). Monitor the child's lipid profile in order to minimize complication (Bhojwani, 2015). Avoid vitamin/mineral supplementing during treatment due to controversies (Eldridgre, 2004).

\subsection{Nutrition Recommendations to Be Considered for Cancer Survivors}

1) Focusing on diet: follow a well-balanced diet that rich in fruits and vegetables. Survivors focus have to be on both the quality and quantity if the food eaten, focus on the nutrient dese foods. Reduce their intake of highly processed meats, refined carbohydrates, saturated fat and Trans fat. Moreover, achieving a healthy life style and behaivor (Eldridgre, 2004).

2) Managing weight: considering that these survivors follow a very restricted diet thought their treatment period they are more likely to think just about eating what they crave for after therapy completion, thus they are highly vulnerable to be obese and overweight which may add up to their chronic complications later on. For that reason, they are encouraged to maintain a healthy body weight and engage in physical activity; minimum of $60 \mathrm{mn} /$ day; that will help in their weight maintenance from one side and increase their FFM plus BMD on the other side (Tylavsky, 2013).

3) Supplementing: vitamin/mineral supplements are considered safe at this point but it is not mandatory unless the cancer survivor is deficient. Hence a frequent visit to the hospital or lap to assess their levels is important. For instance, evaluating vitamin D, calcium, iron, magnesium, folate...etc. is significant for confirming their balanced energy and nutrient intake, and proper growth, and good immune function (Eldridgre, 2004).

4) Regular hospital checkup: in order to follow up their overall health status and make sure everything is ok.

\section{Conclusions}

Pediatrics are not just a spitting image of young adults, they are categorized into several different age groups (neonates, infants, young children, and adolescents). Each group is at a different developmental and growth stage with varied nutrition and energy requirement. Adequate and appropriate individualized nutritional management and assessment are critical to pediatrics with ALL in order to improve their tolerance of treatment, to diminish nutrition impact symptoms and toxicity, and to improve the quality of life as well as maximizing survival rate. Current lack of clear definitive based guidelines addressing the optimal nutrition prescription regarding energy, nutrition, physical activity, and the use of dietary supplements during and after therapy presents a setback to overcome malnutrition. Therefore, encouraging the participation of patients in clinical trials directed towards the nutritional aspects across a wide country range might help in establishing these guidelines and use them a milestone in ALL treatment and follow up.

\section{Declaration}

The authors have no financial support or funds to declare.

\section{REFERENCES}

[1] Bhojwani D, Darbandi R, Pie D, Ramsey LB, Chemaitilly W, Sandlund JT, Cheng C, Relling MV, Heha S, Metzger ML. Severe hypertriglyceridemia during therapy for childhood acute lymphoblastic leukemia. Eur J Cancer. 2014; 50(15): 2685-94. PubMED PMID: 25087182.

[2] Eldridgre B, MS, RD, LD. Medical nutrition therapy for cancer prevention, treatment, and recovery. In: Mahan LK, EscottStump S. Krause's food, nutrition, and diet therapy. Saunders; 
2004. p. $997-1004$.

[3] Han Y, Zhang F, Wang J, Zhu Y, Dia J, Bu Y, Yang Q, Xiao $Y$, Sun $X$. Application of glutamine-enriched therapy in childhood acute ly mphoblastic leukemia. Nutr J. 2016; 15(1): 65. PubMed PMID: 27401338.

[4] Kurylowicz A, Bednarczuk T, Nauman J. The influence of vitamin D deficiency on cancers and autoimmune disease development. Endokry nol Pol. 2007; 58(2): 140-52. PubMED PMID: 17578830.

[5] Owens JL, Hanson SJ, McArthur JA, Mikhailov TA. The need for evidence based nutritional guidelines for pediatric acute lymphoblastic leukemia patients: acute and long-term following treatment. Nutrients. 2013; 5(11): 4333-46. PubMed PMID: 24177709.

[6] PDQ. Childhood acute lymphoblastic leukemia treatment (PDQ ${ }^{\circledR)}$ ): pateints version. PDQ Pediattric Treatment Editorial Board. 2016. PubMED PMID: 26389385.

[7] PDQ. Childhood acute lymphoblastic leukemia treatment (PDQ $\left.{ }^{\circledR}\right)$ : health professional version. PDQ Pediattric Treatment Editorial Board. 2016. PubMED PMID: 26389206.

[8] Selwood K, Ward E, Gibson F. Aseessment and management of nutritional challenges in children's cancer care: a survey of current practice in the United Kingdom. Eur J Oncol Nurs. 2010; 14(5): 439-46. PubMED PMID: 20580897.

[9] Shams-White M, Kelly MJ, Gilhooly C, Liu S, Must A, Parsons SK, Saltzman E, Zhang FF. Food craving and obesity in survivors of pediatric ALL and ly mphoma. Appetite. 2016; 96:1-6. PubMED PMID: 26327446.

[10] Sgarbieri UR, Fisberg M, Tone LG, Latorre Mdo R. Nutritional assessment and serum zinc and copper concentration among children with acute lymphoblastic leukemia: a longitudinal study. Sao Paulo Med J. 2006; 124(6): 316-20. PubMED PMID: 17322951.

[11] Tandon S, Moulik NR, Kumar A, Mahdi AA, Kumar A. Effect of pre-treatment nutritional status, folate and vitamin B12 levels on induction chemotherapy in children with acute ly mphoblastic leukemia. Indian Pediatr. 2015; 52(5):385-9. PubMed PMID: 26061923.

[12] Zhang FF, Liu S, Chung M, Kelly MJ. Growth patterns during and after treatment in patients with pediatric ALL: A metaanalysis. Pediatr Blood Cancer. 2015; 62(8): 1452-60. PubMED PMID: 25808413.

[13] Zhang FF, Roberts SB, Parsons SK, Must A, Kelly MJ, Wong WW, Saltzman E. Low levels of energy expenditure in childhood cancer survivors: implication for obesity prevention. J Pediatr Hematol Oncol. 2015; 37(3): 232-6. PubMED PMID: 25197775. 